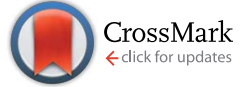

Cite this: Chem. Sci., 2014, 5, 4278

\title{
Well plate circular dichroism reader for the rapid determination of enantiomeric excess $\uparrow$
}

\begin{abstract}
P. Metola, ${ }^{a}$ S. M. Nichols, ${ }^{b}$ B. Kahr ${ }^{\star b}$ and E. V. Anslyn ${ }^{\star a}$
Circular dichroism (CD) spectropolarimeters typically employ one photoelastic modulator. However, spectropolarimeters employing two or even four modulators are more versatile and can be used to subvert common measurement errors arising from imperfectly isotropic samples or sample holders. Small linear anisotropies that can cause large errors in CD measurement can be associated with multiwell sample holders. Thus, high-throughput $C D$ analyses in multi-well plates have not yet been demonstrated. One such application is the determination of enantiomeric excess of a library of reaction products. Herein, a spectropolarimeter employing four photoelastic modulators and a translation stage was used to determine the enantiomeric excess of a family of chiral amine complexes much more rapidly than could be achieved with a robotic fluid injection system. These experiments are proof of concept for high-throughput CD analysis. In practice, commercially available glass bottomed well plates are sufficiently strain free that a simple instrument with just one photoelastic modulator and a vertical optical train should be able to deliver the CD without special considerations given herein. On the other hand, polystyrene well plates cannot be used in this way.
\end{abstract}

Received 3rd June 2014

Accepted 24th July 2014

DOI: $10.1039 / c 4 s c 01641 f$

www.rsc.org/chemicalscience

into the realm of HTS, a boon to the synthesis community because a growing number of ee assays involve chiroptical measurement. ${ }^{29}$ For example, the groups of Anslyn and Wolf have developed and tested CD methods for the analysis of chiral analytes including amino acids, ${ }^{30}$ alcohols, ${ }^{31}$ amino alcohols ${ }^{32,33}$ carboxylates $^{34}$ and amines. ${ }^{35-38}$ Biological/biochemical sciences may benefit likewise. CD spectroscopy is sensitive to the conformational analysis of peptides, proteins, and nucleic acids. ${ }^{39,40} \mathrm{CD}$ can also be used to study dynamic processes such as binding interactions between a macromolecule and small molecule drugs, dyes, guests, and ligands..$^{39-41}$

Manufacturers of CD spectropolarimeters have attempted to streamline the acquisition of data by designing auto-samplers or robotic injectors, ${ }^{42}$ but they only marginally speed measurement; CD "robots" are not true plate readers but automated flow injectors, drawing each sample and injecting it sequentially. Moreover, they can be sensitive to non-aqueous media, malfunctioning when handling common organic solvents. ${ }^{43}$

Why is a HTS-CD spectrometer not currently available? Presumably, this is because multi-well plates are not necessarily optically isotropic. They can be burdened with strain linear birefringence (LB), i.e. refractive index anisotropy. Parasitic LB has plagued measurements of the $\mathrm{CD}$ of anisotropic media for decades. ${ }^{44-50}$ Moreover, menisci - non-normal interfaces - in well plates can introduce Fresnel linear dichroism (LD) into the optical system. LB and LD can be much larger than circular birefringence ( $\mathrm{CB}$ or optical rotation) and $\mathrm{CD}$. The minor chiroptical perturbation to the polarization state of light in anisotropic media can be of the same order of magnitude as

\footnotetext{
${ }^{a}$ Department of Chemistry, The University of Texas at Austin, Austin, Texas, 78712, USA. E-mail: anslyn@austin.utexas.edu

${ }^{b}$ Department of Chemistry and Molecular Design Institute, New York University, New York, New York 10003, USA. E-mail: bart.kahr@nyu.edu

$\dagger$ Electronic supplementary information (ESI) available: Details of experimenta conditions, CD and ee calculations, Mueller matrix computations and imaging of polystyrene well plate anisotropy. See DOI: 10.1039/c4sc01641f
} 
parasitic ellipticities from imperfect samples, containers, and optical components.

\section{Experimental strategy}

\section{Instrument design}

CD spectropolarimeters typically modulate between left and right circular polarization states by using a photoelastic modulator (PEM) placed before the sample. The differential transmission is recorded. But the measured $\mathrm{CD}$ can be grossly affected by the admixture of linear polarization in the input light and its coupling to linear anisotropies of the medium (in solid samples) or the container. Multiple photoelastic modulators (PEMs) provide a strategy for separating and eliminating contributions to the change in the polarization of light due to parasitic linear anisotropies that have traditionally given rise to artifactual chiral signatures. ${ }^{51,52}$

Most polarization techniques rely on measuring particular elements of the sample Mueller matrix, the linear operator that contains all of the information about the polarization transforming properties of an analyte. ${ }^{53}$ Increasingly complex samples spread the desired optical effects over all 16 elements of the Mueller matrix. ${ }^{45}$ In 2011, we introduced a polarimeter using four PEMs (4-PEM spectropolarimeter or simply "4-PEM" in brief) that measures simultaneously all Mueller matrix elements without reconfiguration, thus avoiding errors associated with moving optical components. ${ }^{51,52} \mathrm{~A}$ schematic is shown in Fig. 1. This instrument is fast, sensitive, and accurate. It will be used here as a high-speed automated plate reader.

In our instrument, light from a Xe arc lamp coupled to a monochromator ( $290 \mathrm{~nm}$ to $850 \mathrm{~nm}$ ) was normally incident on the sample. The polarization state generator and the polarizer state analyzer are each composed of a linear polarizer and two PEMs (Hinds Instruments) operating at a different frequencies. The PEMs were set at relative orientations of $\pm 45^{\circ}$. The advantage of this setup over other Mueller matrix polarimeters is that measurements are obtained without any moving parts. The transmitted light was detected with a photomultiplier. The 4-PEM measures the 16 parameters of a normalized Mueller matrix at each wavelength.

A 96-well glass bottom plate (In Vitro Scientific, P96-1.5H-N, with a MatTek Corporation top cover glass, PCS10872-1.0) was mounted on two linear actuators (Newport) with a range of only three wells in the vertical and horizontal directions, thus only a $3 \times 3$ block of wells could be measured without repositioning

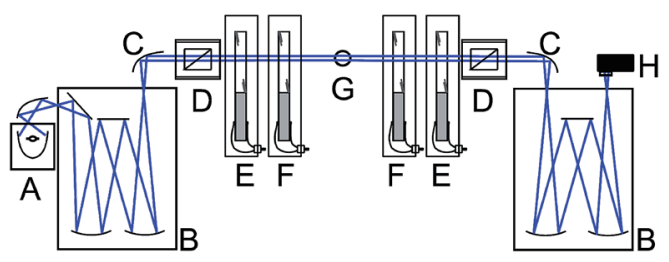

Fig. 1 Four photoelastic modulator (4-PEM) spectropolarimeter. (A) Light source, (B) monochromators, (C) mirrors, (D) linear polarizers, ( $E$ and F) photoelastic modulators set at $0^{\circ}$ (E) and $45^{\circ}$ (F). (G) Sample holder, $(\mathrm{H})$ photomultiplier tube.

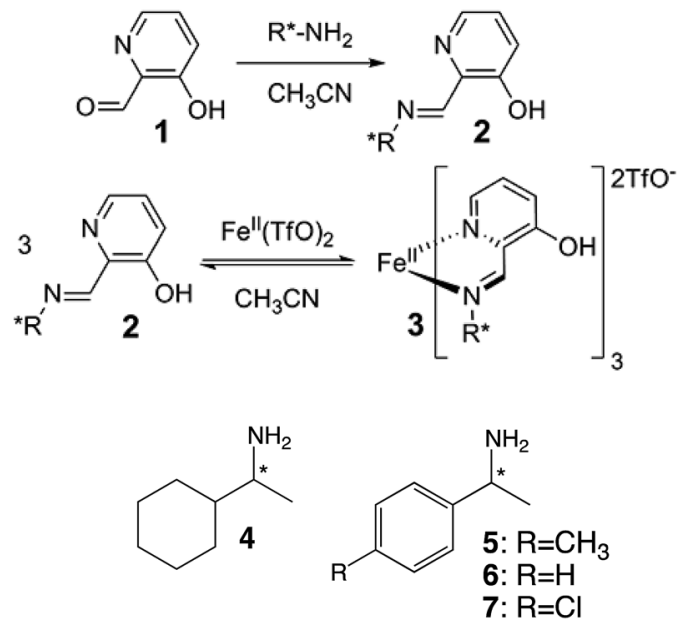

Scheme 1 Two-step assembly that leads to the CD-active $\mathrm{Fe}^{(\mathrm{III}}$ complex used to report the ee of chiral amines 4-7.

the plate. The cover glass was secured with elastic bands because our polarimeter has a horizontal light path. There are no obstacles to building a comparable instrument with a vertical optical train and a high-speed translation stage outfitted for well plates. But, for proof of concept here, we used the instrument currently in operation (in fact, two PEMs or even one will suffice). With average reading times of about two seconds for a single wavelength and around 30 seconds for a $120 \mathrm{~nm}$-wide scan per sample, the rate of CD analysis can truly be called high-throughput. Single ellipticity values (as used herein) can be read for the entire plate in less than three minutes.

\section{Chemical system}

With the goal of testing the performance of a multiple PEM polarimeter in HTS measurements, we chose a validated CD assay for ee of $\alpha$-chiral amines. ${ }^{35}$ Aldehyde 1 reacts with the a chiral amine (4-7) to give a bidentate imine 2 . Fe(II), in turn, binds three equivalents of that imine to give several helical octahedral complexes represented as $\mathbf{3}$, whose handedness is dictated by the chirality of the amine (Scheme 1). We determined the enantioenrichment of amine 4-7 by measuring CD in the strong metal-to-ligand charge transfer bands that arise upon formation of complex $3 .^{35}$ This reaction is fast, simple, relies on commercially available reagents and can be analyzed in situ.

\section{Results and discussion}

\section{Mueller matrix polarimetry}

A Mueller matrix is a real valued $4 \times 4$ matrix that represents an optical element or sample. Stokes-Mueller calculus tracks the transformation of the state of polarization of light passing through the 4-PEM and the sample.

The Mueller matrix of an optically active solution, $\left(M_{\mathrm{sol}}\right)$ in (1), has only two parameters where $\alpha=\mathrm{CD}, \beta=\mathrm{CB}$, and $\alpha^{2}+\beta^{2}+$ $\gamma^{2}=1$. It has been shown previously that a Mueller matrix 
which introduces small, parasitic linear anisotropies (LB and LD less than $\sim 0.5 \mathrm{rad}$ ) and negligible depolarization can be expressed as the explicit matrix $\left(M_{\text {anis }}\right)$ in $(1) .{ }^{54}$ Here, $\mathrm{LB}^{\prime}$ and $\mathrm{LD}^{\prime}$ refer to a set of orthogonal axes at $\pm 45^{\circ}$ with respect to the instrument coordinate system.

In the absence of linear anisotropies, CD can be taken directly from either the $M_{03}$ or $M_{30}$ elements. Commercial CD spectrometers typically measure $M_{03}$ using a single PEM positioned before the sample. However, even small ellipticities introduced by the sample holder will perturb the measured Mueller matrix. To evaluate the Mueller matrix with anisotropy introduced into sample holder before or after the sample, i.e. closer to or further from the light source, we analyzed the respective matrix products $M_{\mathrm{sol}} M_{\mathrm{anis}}$ or $M_{\mathrm{anis}} M_{\mathrm{sol}}$. from the artifact-free CD that result from measurement of only $M_{03}$ or $M_{30}$ in the presence of modest anisotropy.

We emulated a well plate measurement of a $0.02 \mathrm{M} \mathrm{Cu(II)-D-}$ tartrate solution in a quartz cuvette placed just before a transparent birefringent material (a piece of adhesive tape). The complete $M$ was measured with the 4-PEM. $M_{03}$ and $M_{30}$ by themselves did not match the CD measured directly in the glass cuvette without the interfering linearly birefringent tape. But, $\left(M_{03}+M_{30}\right) /\left(1+M_{33}\right)$ matched quite well.

With the In Vitro Scientific glass bottomed well plates, $M_{03}$ and $M_{30}$ were equal and either could be taken as the CD. This implies that glass bottomed well plates, at least from this manufacturer, can be used along with a single PEM. That is a HTS CD instrument could well be fashioned from a commercial device with a vertical optical train and a high speed scanning

$$
\begin{aligned}
M_{\text {sol }} & =\left[\begin{array}{cccc}
1 & 0 & 0 & \alpha \\
0 & \gamma & \beta & 0 \\
0 & -\beta & \gamma & 0 \\
\alpha & 0 & 0 & 1
\end{array}\right], \\
M_{\text {anis }} & =\left(\begin{array}{cccc}
1+1 / 2\left(\mathrm{LD}^{2}+\mathrm{LD}^{-2}\right) & -\mathrm{LD} & -\mathrm{LD}^{\prime} & 1 / 2\left(\mathrm{LBLD}^{\prime}+\mathrm{LB}^{\prime} \mathrm{LD}\right) \\
-\mathrm{LD} & 1+1 / 2\left(\mathrm{LD}^{2}+\mathrm{LB}^{\prime 2}\right) & 1 / 2\left(\mathrm{LBLB}^{\prime}+\mathrm{LDLD}^{\prime}\right) & \mathrm{LB}^{\prime} \\
-\mathrm{LD}^{\prime} & 1 / 2\left(\mathrm{LBLB}^{\prime}+\mathrm{LDLD}^{\prime}\right) & 1+1 / 2\left(\mathrm{LD}^{\prime 2}+\mathrm{LB}^{2}\right) & -\mathrm{LB}^{\prime} \\
-1 / 2\left(\mathrm{LBLD}^{\prime}+\mathrm{LB}^{\prime} \mathrm{LD}\right) & -\mathrm{LB}^{\prime} & \mathrm{LB} & 1-1 / 2\left(\mathrm{LB}^{2}+\mathrm{LB}^{\prime 2}\right)
\end{array}\right)
\end{aligned}
$$

As explained in the ESI, $\uparrow$ regardless of the sample position, analysis of the matrix products shows that CD can be approximated from the elements of measured Mueller matrix as CD = $\left(M_{03}+M_{30}\right) /\left(1+M_{33}\right)$. For very small linear anisotropies, $M_{33}$ approaches a value of one, and the correction for CD approaches the simple average of $M_{03}$ and $M_{30}$. For large linear anisotropies, $M_{03}$ and $M_{30}$ will be very different from one another, and the correction fails. Fig. 2 illustrates the deviations

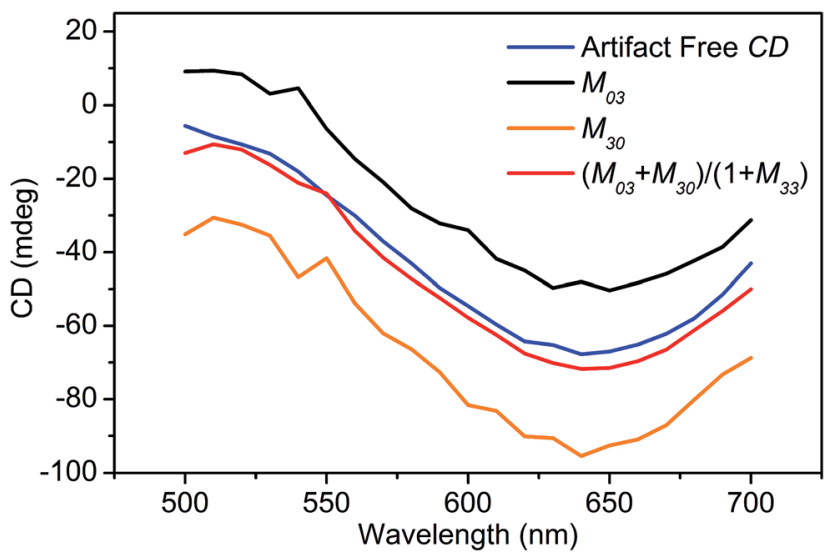

Fig. 2 CD errors when using only one PEM. A $0.02 \mathrm{M}$ solution of $\mathrm{Cu}(॥)$ tartrate measured without (artifact-free $C D$ ) and with (all other spectra) a birefringent piece of adhesive tape placed after the solution cuvette using the 4-PEM. stage of the type already adapted to simple UV-vis HTS. However, there is no direct way to verify the CD measurements with a single PEM, while for two (or in this case four) PEMs, $M_{03}$, $M_{30}$, and $M_{33}$ can be monitored simultaneously to increase data reliability. Additionally, two PEMs deliver other elements of the Mueller matrix that can be used to estimate the magnitude of the linear anisotropies. ${ }^{55,56}$ We caution that polystyrene well plates are unsuitable for the measurement of $\mathrm{CD}$ as the perturbing LB is much too large. Such a plate was analyzed with the 4-PEM and the heterogeneities in the optical properties were captured with an imaging Mueller matrix microscope described previously (see ESI $\dagger$ ). ${ }^{57}$

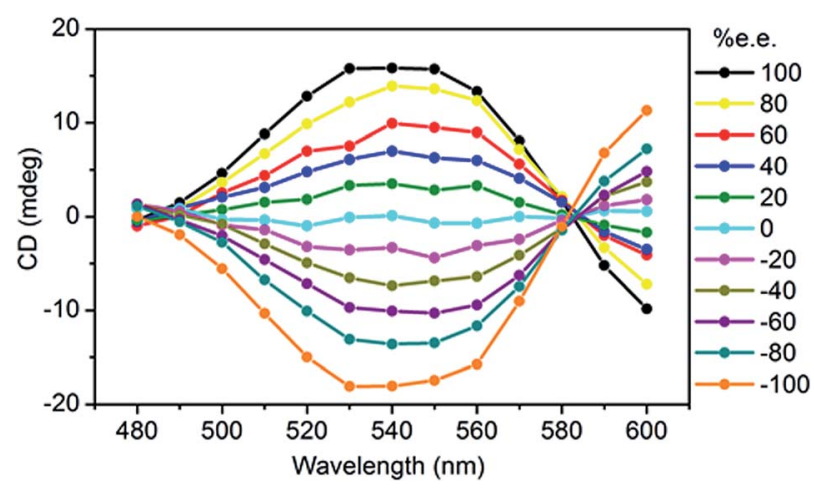

Fig. 3 CD spectra for the assembly with 4 


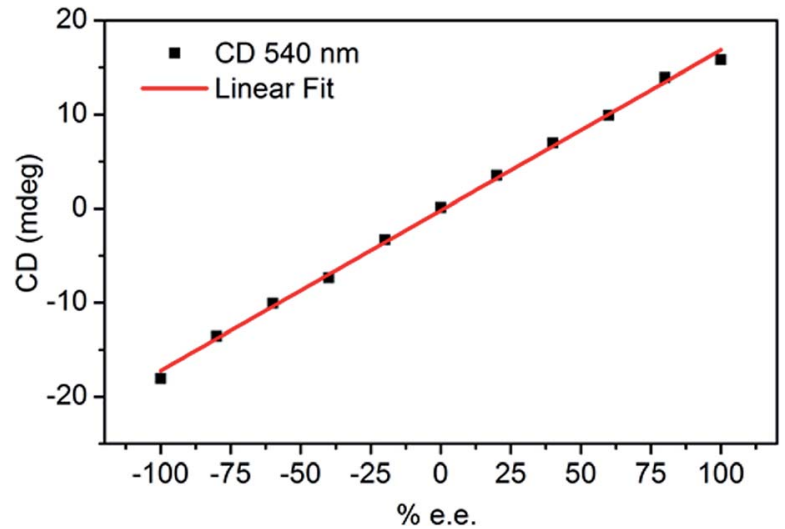

Fig. 4 Calibration curve for the assembly with 4 at $540 \mathrm{~nm}$.

\section{Enantiomeric excess determination in well plates}

Amines 4-7 in Fe complexes in Scheme 1 were analyzed by the 4PEM. CD spectra were measured (Fig. 3) for solutions spanning the enantiomeric composition between $100 \% R$ configuration and $100 \% S$ configuration. A calibration curve was built from $M_{03}$ values (Fig. 4) and fit to a line in the case of 4 . The $R^{2}$ value was 0.998 .

While the calibration curve in Fig. 4 is a straight line for $\mathbf{4}$, such curves not generally linear because the CD represents mixtures of stereoisomers at intermediate concentrations. The curves in the ESI $\dagger$ are generally sigmoidal and fit to $3^{\text {rd }}$ order polynomials. See ESI. $\dagger$

Once the calibration curves that relate CD signal intensity to ee for each amine were obtained, a number of "unknown" samples were analyzed from new stock solutions of products. The CD values were converted to values of ee using the calibration curves previously established. The calculated values of ee were compared to the actual values of the solutions measured. The ee errors ranged between $|5-9| \%$. These are errors in chemistry (weighing reagents and volumes) and are consistent with those found when measuring such samples individually and fall within the range of accuracy that is expected for a HTS method. ${ }^{28}$

\section{Conclusions}

With a spectropolarimeter carrying more than one photoelastic modulator, $C D$ was measured rapidly in a multi-well plate format. The technology has been tested with an existing sensing ensemble and the results are consistent with previous data. There should be few obstacles to HTS-CD in high quality well plates with glass interfaces.

\section{Acknowledgements}

AVE gratefully acknowledges the financial support from the National Institutes of Health (GM077437) and the Welch Foundation (F-1151). BK thanks the National Science Foundation (DMR-1105000) and SMN is grateful for a National Science Foundation Predoctoral Fellowship (DGE-12342536).

\section{Notes and references}

1 G. Alves, A. Fortuna and A. Falcao, Trends Chromatogr., 2008, 4, 1-10.

2 J. P. Stambuli and J. F. Hartwig, Curr. Opin. Chem. Biol., 2003, 7(3), 420-426.

3 K. D. Shimizu, M. L. Snapper and A. H. Hoveyda, Chem.-Eur. J., 1998, 4(10), 1885-1889.

4 S. H. Shabbir, C. J. Regan and E. V. Anslyn, Proc. Natl. Acad. Sci. U. S. A., 2009, 106(26), 10487-10492.

5 M. T. Reetz, Angew. Chem., Int. Ed., 2002, 41(8), 1335-1338.

6 J. F. Traverse and M. L. Snapper, Drug Discovery Today, 2002, 7(19), 1002-1012.

7 C. Jäkel and R. Paciello, Chem. Rev., 2006, 106(7), 2912-2942.

8 C. Gennari and U. Piarulli, Chem. Rev., 2003, 103(8), 30713100 .

9 D. Wahler and J.-L. Reymond, Curr. Opin. Biotechnol., 2001, 12(6), 535-544.

10 C. Gennari, S. Ceccarelli, U. Piarulli, C. A. G. N. Montalbetti and R. F. W. Jackson, J. Org. Chem., 1998, 63(16), 53125313.

11 J. G. de Vries and L. Lefort, Chem.-Eur. J., 2006, 12(18), 47224734.

12 K. Ding, Chem. Commun., 2008, 8, 909-921.

13 L. Lefort, J. A. F. Boogers, A. H. M. de Vries and J. G. de Vries, Top. Catal., 2006, 40(1-4), 185-191.

14 A. J. Minnaard, B. L. Feringa, L. Lefort and J. G. de Vries, Acc. Chem. Res., 2007, 40(12), 1267-1277.

15 W. Seiche and B. Breit, in Phosphorus Ligands in Asymmetric Catalysis: Synthesis and Applications, ed. A. Börner, WileyVCH, Weinheim, 2008.

16 K. Sakthivel, W. Notz, T. Bui and C. F. Barbas III, J. Am. Chem. Soc., 2001, 123(22), 5260-5267.

17 L. Lefort, J. A. F. Boogers, A. H. M. de Vries and J. G. de Vries, Org. Lett., 2004, 6(11), 1733-1735.

18 B. L. Feringa, Acc. Chem. Res., 2000, 33(6), 346-353.

19 O. Pàmies and M. Diéguez, Chem.-Eur. J., 2008, 14(3), 944-960.

20 P. P. Pescarmona, J. C. Van der Waal, I. E. Maxwell and

T. Maschmeyer, Catal. Lett., 1999, 63(1-2), 1-11.

21 R. H. Crabtree, Chem. Commun., 1999, 1611-1616.

22 D. G. Powers and D. L. Coffen, Drug Discovery Today, 1999, 4(8), 377-383.

23 R. B. C. Jagt, P. Y. Toullec, E. P. Schudde, J. G. De Vries, B. L. Feringa and A. J. Minnaard, J. Comb. Chem., 2007, 9(3), 407-414.

24 T. M. Tarasow, S. L. Tarasow and B. E. Eaton, Nature, 1997, 389(6646), 54-57.

25 M. T. Reetz, K. M. Kuhling, S. Wilensek, H. Husmann, U. W. Hausig and M. Hermes, Catal. Today, 2001, 67(4), 389-396.

26 D. C. Pregibon, M. Toner and P. S. Doyle, Science, 2007, 315(5817), 1393-1396.

27 C. Boozer, G. Kim, S. Cong, H. Guan and T. Londergan, Curr. Opin. Biotech., 2006, $17(4)$, 400-405.

28 D. Leung and E. V. Anslyn, J. Am. Chem. Soc., 2008, 130(37), 12328-12333. 
29 C. Wolf and K. W. Bentley, Chem. Soc. Rev., 2013, 42(12), 5408-5424.

30 M. W. Ghosn and C. Wolf, Tetrahedron, 2010, 66(23), 39893994.

31 L. You, J. S. Berman and E. V. Anslyn, Nat. Chem., 2011, 3(12), 943-948.

32 M. W. Ghosn and C. Wolf, J. Am. Chem. Soc., 2009, 131(45), 16360-16361.

33 D. P. Iwaniuk, K. W. Bentley and C. Wolf, Chirality, 2012, 24(7), 584-589.

34 L. A. Joyce, M. S. Maynor, J. M. Dragna, G. M. da Cruz, V. M. Lynch, J. W. Canary and E. V. Anslyn, J. Am. Chem. Soc., 2011, 133(34), 13746-13752.

35 J. M. Dragna, G. Pescitelli, L. Tran, V. M. Lynch, E. V. Anslyn and L. Di Bari, J. Am. Chem. Soc., 2012, 134(9), 4398-4407.

36 P. Metola, E. V. Anslyn, T. D. James and S. D. Bull, Chem. Sci., 2011, 3(1), 156-161.

37 M. W. Ghosn and C. Wolf, Tetrahedron, 2011, 67(36), 67996803.

38 D. P. Iwaniuk and C. Wolf, Org. Lett., 2011, 13(10), 2602-2605.

39 N. Berova, K. Nakanishi and R. W. Woody, Circular dichroism: principles and applications, Wiley-VCH, New York, 2000.

40 N. Purdie and H. G. Brittain, Analytical applications of circular dichroism, Elsevier, 1993.

41 M. Eriksson and B. Nordén, Methods Enzymol., 2001, 340, 68-98. 42 Jasco ASU-800 Autosampler.

43 In our experience, the tubing is not compatible with acetonitrile or halogenated solvents, and the robotic handler rapidly plugs. The threshold for concentration tolerated by the plastic tubes before clogging occurs is low.

44 K. W. Hipps and G. A. Crosby, J. Phys. Chem., 1979, 83(5), 555-562.

45 J. Schellman and H. P. Jensen, Chem. Rev., 1987, 87(6), 13591399.

46 J. Michl and E. W. Thulstrup, Spectroscopy with Polarized Light, VCH, New York, 1986.

47 R. Kuroda, T. Harada and Y. Shindo, Rev. Sci. Instrum., 2001, 72(10), 3802-3810.

48 M. F. Maestre, G. C. Salzman, R. A. Tobey and C. Bustamante, Biochemistry, 1985, 24(19), 5152-5157.

49 J. R. L. Moxon and A. R. Renshaw, J. Phys.: Condens. Matter, 1990, 2, 6807-6836.

50 M. Kremers and H. Meekes, J. Phys. D: Appl. Phys., 1995, 28(6), 1212-1224.

51 O. Arteaga, J. Freudenthal, B. Wang, S. Nichols and B. Kahr, Chim. Oggi, 2012, 30, 31-36.

52 O. Arteaga, J. Freudenthal, B. Wang and B. Kahr, Appl. Opt., 2012, 51(28), 6805-6817.

53 D. H. Goldstein, Polarized Light, 3rd edn, CRC Press, Boca Raton, 2010.

54 O. Arteaga, Ph.D. thesis, University of Barcelona, 2011.

55 G. E. Jellison Jr and F. A. Modine, Appl. Opt., 1997, 36(31), 8184-8189.

56 G. E. Jellison Jr and F. A. Modine, Appl. Opt., 1997, 36(31), 8190-8198.

57 A. G. Shtukenberg, J. Freudenthal and B. Kahr, J. Am. Chem. Soc., 2010, 132(39), 9341-9349. 